\title{
A Study on Radiation Damage in Large Size LSO and LYSO Crystal Samples
}

\author{
Jianming Chen Member, IEEE, Rihua Mao, Liyuan Zhang Member, IEEE and Ren-yuan Zhu Senior Member, IEEE
}

\begin{abstract}
This paper presents a study on radiation damage effect in large size $\left(2.5 \times 2.5 \times 20 \mathrm{~cm}^{3}\right)$ LSO and LYSO crystals. Optical and scintillation properties, including the longitudinal transmittance and emission spectra, the light output and light response uniformity with PMT and APD readout, are measured before and after $\gamma$-ray irradiations with integrated dosage up to $10^{6} \mathrm{rad}$ for three LSO and LYSO samples from different vendors. It was found that the recovery of radiation damage under room temperature is negligible, indicating that radiation damage in LSO and LYSO crystals are not dose rate dependent. It was also found that the overall radiation damage in LSO and LYSO crystals is small as compared to other crystal scintillators commonly used in high energy and nuclear physics experiments.
\end{abstract}

Index Terms-Lutetium Oxyorthosilicate; Lutetium Yttrium Oxyorthosilicate; Crystal; Scintillator; Transmission; Emission; Light Output; Radiation Damage.

\section{INTRODUCTION}

I $\mathrm{N}$ the last decade, mass production capabilities of Gadolinium Orthosilicate $\left(\mathrm{Gd}_{2} \mathrm{SiO}_{5}, \mathrm{GSO}\right)$ [1], lutetium oxyorthosilicate $\left(\mathrm{Lu}_{2} \mathrm{SiO}_{5}, \mathrm{LSO}\right)$ [2] and lutetium-yttrium oxyorthosilicate $\left(\mathrm{Lu}_{2(1-x)} \mathrm{Y}_{2 x} \mathrm{SiO}_{5}\right.$, LYSO) [3], [4] crystals are established for medical industries. Because of their high stopping power and fast bright scintillation, LSO and LYSO crystals have also attracted broad interest in the high energy physics community for future experiments, such as a super B factory [5] and the international linear collider (ILC) [6]. Following our previous studies [7], [8] this report presents a further study on radiation damage in large size LSO and LYSO samples.

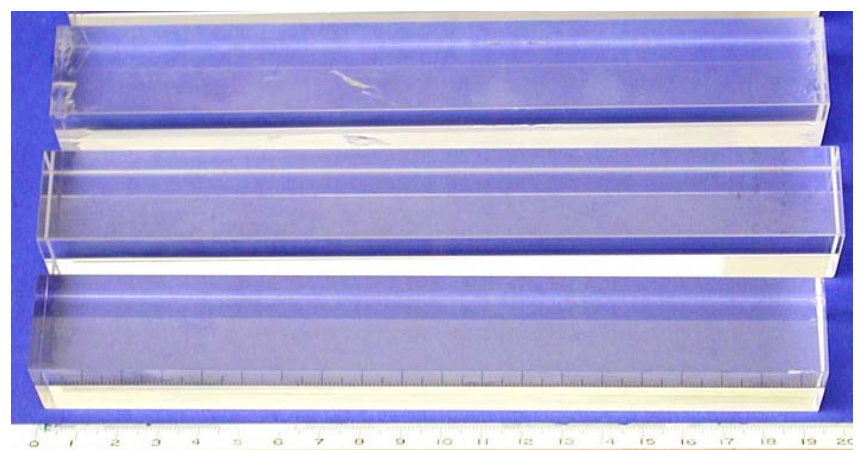

Fig. 1. A photo showing three long $(2.5 \times 2.5 \times 20 \mathrm{~cm})$ crystal samples, from top to bottom: CPI LYSO, SG LYSO and CTI LSO.

This work was supported in part by the U.S. Department of Energy Grant No. DE-FG03-92-ER40701.

The authors are with the California Institute of Technology, Pasadena, CA 91125, USA (E-mail: zhu@hep.caltech.edu).
Fig. 1 is a photo showing three long crystal samples with dimension of $2.5 \times 2.5 \times 20 \mathrm{~cm}$. They are, from top to bottom, an LYSO samples from Crystal Photonics, Inc. (CPI), an LYSO sample from Saint-Gobain Ceramics \& Plastics, Inc. (SaintGobain) and an LSO sample from CTI Molecular Imaging (CTI). It is noted that the CPI LYSO sample has chips at corner and surface since CPI does not have adequate polishing and treatment facilities for such large size samples [9].

According to the manufactures, the yttrium content is about $5 \%$ for the CPI LYSO [9] and about 10\% for the Saint-Gobain LYSO [10]. The nominal cerium doping level is $0.2 \%$ for the CTI LSO [11] and the CPI LYSO [9], and is less than $1 \%$ for the Saint-Gobain LYSO [10]. The actual cerium concentration in these crystals, however, would be less than the nominal value and its distribution along sample's axis also varies because of the segregation. The technical nature of long crystal's ends, such as the seed or tail in growth, are not provided by the manufactures. All surfaces of these samples are polished.

TABLE I

IRRADIATION TIME AND THE INTEGRATED DOSAGE

\begin{tabular}{ccccccc}
\hline \hline Dosage (rad) & 10 & $10^{2}$ & $10^{3}$ & $10^{4}$ & $10^{5}$ & $10^{6}$ \\
$\gamma$-ray Source & ${ }^{60} \mathrm{Co}$ & ${ }^{60} \mathrm{Co}$ & ${ }^{137} \mathrm{Cs}$ & ${ }^{137} \mathrm{Cs}$ & ${ }^{137} \mathrm{Cs}$ & ${ }^{137} \mathrm{Cs}$ \\
Dose rate (rad/h) & 2 & 100 & 8,500 & 8,500 & 8,500 & 8,500 \\
Time (hour) & 5 & 0.9 & 0.106 & 1.06 & 10.6 & 106 \\
\hline \hline
\end{tabular}

$\gamma$-ray irradiations were carried out at two irradiation facilities at Caltech: an open 50 curie ${ }^{60} \mathrm{Co}$ source and a closed 2,000 curie ${ }^{137} \mathrm{Cs}$ source. The former provides dose rates of 2 and $100 \mathrm{rad} / \mathrm{h}$ by placing samples at appropriate distances. The later provides a dose rates of $8,500 \mathrm{rad} / \mathrm{h}$ with $5 \%$ uniformity when samples are placed at the center of the irradiation chamber. Irradiations were carried out step by step with integrated dosage of $10,10^{2}, 10^{3}, 10^{4}, 10^{5}$ and $10^{6} \mathrm{rad}$. Table I lists integrated dosage and corresponding irradiation time and dose rate applied to these samples in each step. Optical and scintillation properties, including the longitudinal transmittance and photoluminescence spectra, the light output and light response uniformity with photomultiplier (PMT) and avalanche photodiode (APD) readout, were measured before and 24 hours after each step of irradiations. Samples were kept in the dark at room temperature in the entire experiment to avoid any thermal annealing and optical breaching. 


\section{Damage in Scintillation And Optical Properties}

\section{A. Photoluminescence}

Samples were first irradiated at a dose rate of $8,500 \mathrm{rad} / \mathrm{h}$ for 96 hours. Excitation and photo luminescence spectra were measured using a Hitachi F-4500 fluorescence spectrophotometer before and after irradiation. For the excitation and emission spectra, a UV excitation light was shot to a bare surface of the sample, and the crystal was oriented so that the photoluminescence emission light is not affected by sample's internal absorption.

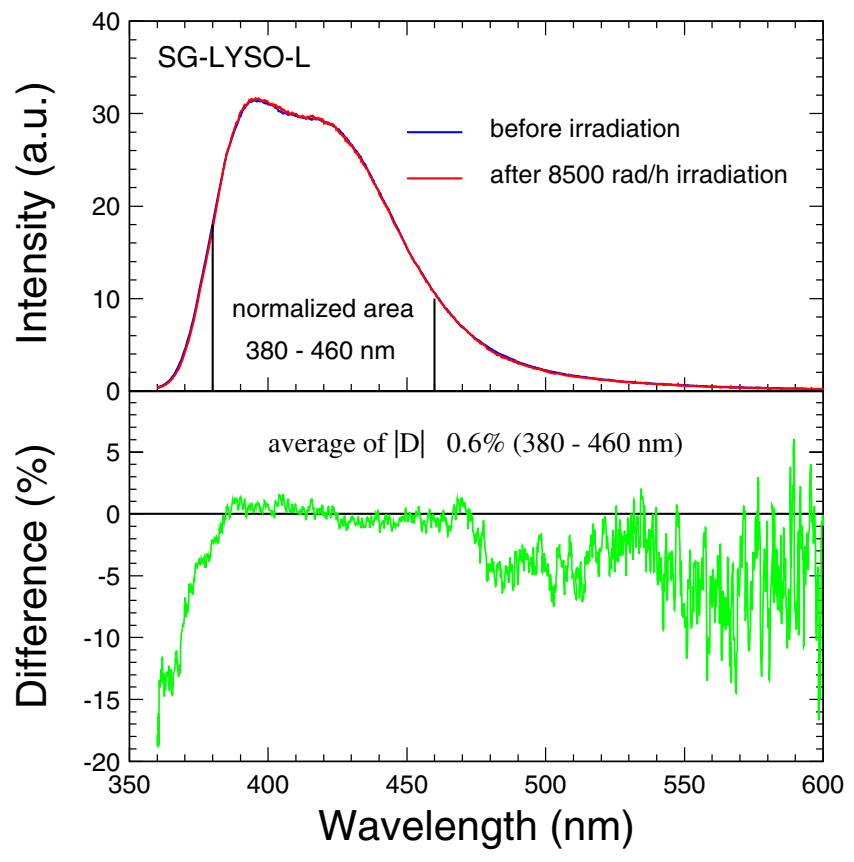

Fig. 2. Top: Photo-luminescence spectra are shown as a function of wavelength for samples SG-LYSO before (blue) and after (red) irradiation. Bottom: The corresponding difference of these spectra is shown as a function of wavelength.

The top plot of Fig. 2 shows the emission spectra of the SGLYSO sample measured before (blue) and (red) after irradiation. To facilitate comparison these spectra were normalized to the area between 380 and $460 \mathrm{~nm}$ under the spectra. The corresponding relative difference is shown in the bottom plot of Fig. 2. The average of the absolute value of the difference between before and after irradiation is found to be $0.6 \%$ in the normalization region, less than the systematic uncertainties of the measurement, which is about $1 \%$. This indicates that $\gamma$-ray irradiation does not affect the scintillation mechanism in LSO and LYSO crystals.

\section{B. Recovery of the Longitudinal Transmittance}

Transmittance spectra were measured by a Perkin Elmer Lambda-950 UV/Vis/NIR spectrophotometer with double beam, double monochromator and an optical bench for these samples before and after irradiation. The systematic uncertainty in repeated measurements is about $0.15 \%$.

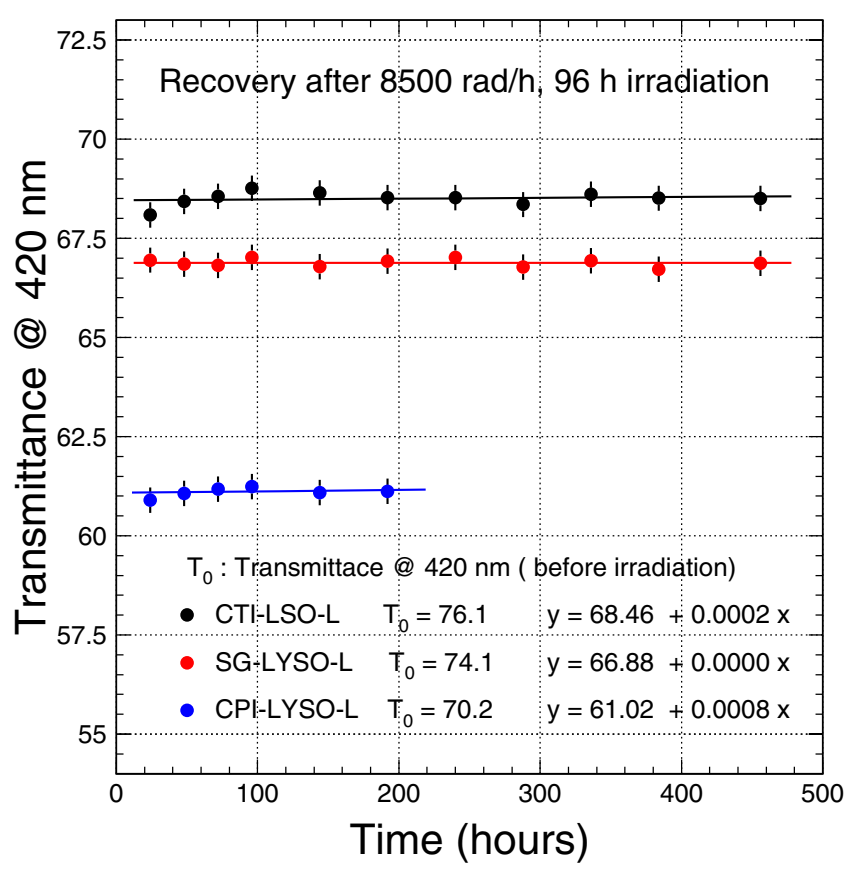

Fig. 3. Values of the longitudinal transmittance at $420 \mathrm{~nm}$ are shown as a function of time for samples CTI-LSO (black), CPI-LYSO (blue) and SG-LYSO (red) after a 96 hour irradiation at $8,500 \mathrm{rad} / \mathrm{h}$.

Fig. 3 shows the longitudinal transmittance values at 420 $\mathrm{nm}$ as a function of the time after irradiation, where $\mathrm{T}_{0}$ is the transmittance before irradiation. Data for each sample are fit to a linear function of time in hours. The slopes are $8 \times 10^{-4}$, $2 \times 10^{-4}$ and 0 respectively for samples CPI, CTI and SaintGobain. The observed recovery of radiation damage in LSO and LYSO samples under room temperature thus is negligible, indicating that the radiation damage in LSO and LYSO crystals is not dose rate dependent [12].

\section{Thermal Annealing and Transmittance Damage}

Since radiation damage in LSO and LYSO crystals shows no dose rate dependence, integrated dosage is used to describe the level of radiation applied. Before irradiations, all samples were annealed at $300^{\circ} \mathrm{C}$ for 10 hours, which eliminates all radiation damage effect in transmittance. After thermal annealing, they were irradiated step by step to integrated dosage of $10,10^{2}$, $10^{3}, 10^{4}, 10^{5}$ and $10^{6} \mathrm{rad}$, as shown in Table I.

Fig. 4 shows the longitudinal transmittance spectra measured for CTI-LSO sample before and after each step of $\gamma$-ray irradiations. Fig. 5 shows the longitudinal transmittance spectra in an expanded scale for these samples before and after each step of $\gamma$-ray irradiations. No difference was observed between LSO and LYSO samples. Also shown in the figure is the numerical values of the emission weighted longitudinal transmittance ( $E W L T)$ before and after each step of irradiations, which is defined as

$$
E W L T=\frac{\int L T(\lambda) \operatorname{Em}(\lambda) d \lambda}{\int E m(\lambda) d \lambda} .
$$




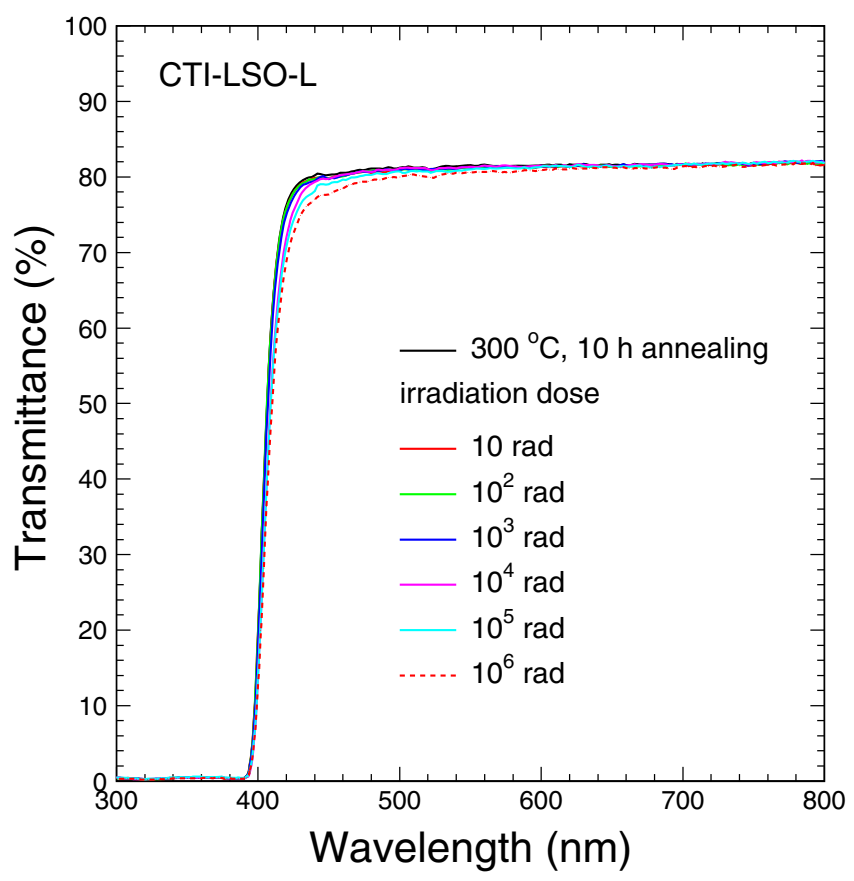

Fig. 4. The longitudinal transmittance spectra are shown as a function of wavelength for CTI-LSO sample after $300^{\circ} \mathrm{C}$ annealing, $10,10^{2}, 10^{3}, 10^{4}$, $10^{5}$ and $10^{6}$ rad irradiations.

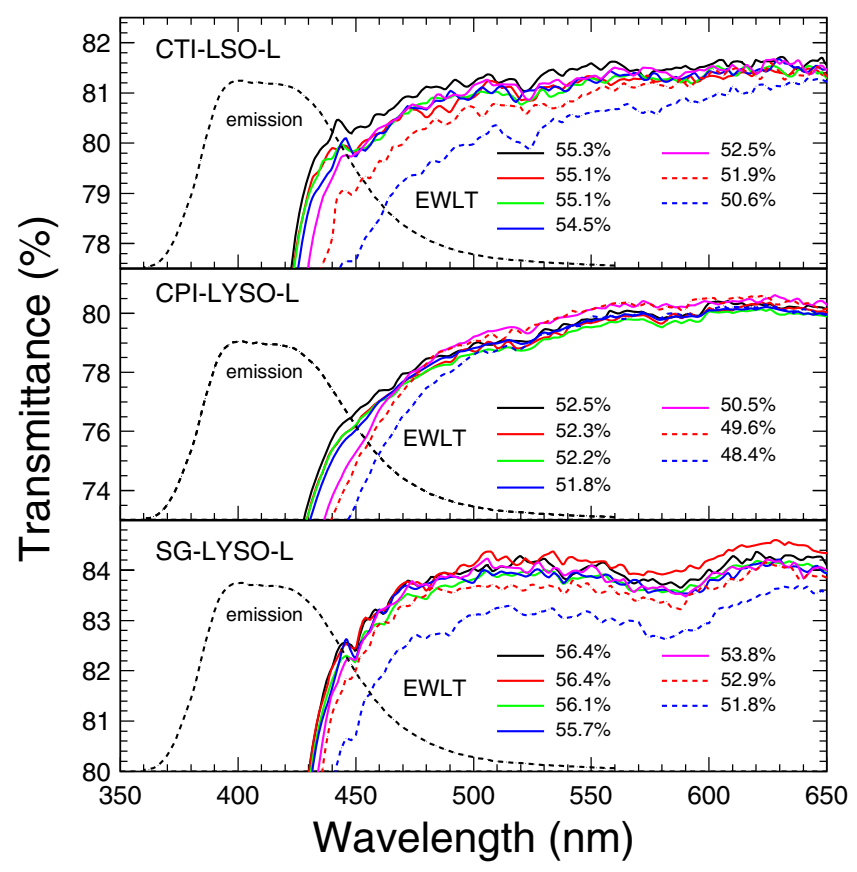

Fig. 5. The transmittance spectra are shown as a function of wavelength in an expanded scale together with the emission spectra for CTI-LSO, CPI-LYSO and SG-LYSO samples before and after each step of irradiations.

A continuous degradation of transmittance was observed. This observation differs from our previous observation [7], where a slight increase of transmittance was observed at low dose irradiation. This difference may be explained by the residual absorption in these samples, which was optically self-bleached by the scintillation light since these samples were not thermally annealed before irradiations in our previous study.

The relative degradation of EWLT after an integrated dosage of $10^{6} \mathrm{rad}$ is $8.5 \%, 7.8 \%$ and $8.2 \%$ respectively for CTILSO, CPI-LYSO and SG-LYSO. The amplitude of the observed $E W L T$ variations is smaller than crystal scintillators commonly used in experimental physics [12], indicating that LSO and LYSO crystals are more radiation resistant.

\section{LIGHT OUTPUT AND RESPONSE UNIFORMITY WITH PMT READOUT}

The light output and decay kinetics were measured using a Photonis XP2254b PMT, which has a multi-alkali photo cathode and a quartz window. To reduce the effect of intrinsic natural radioactivity, a collimated ${ }^{22} \mathrm{Na}$ source was used with a coincidence trigger provided by a $\mathrm{BaF}_{2}$ crystal [8]. The $\gamma$-ray peak was obtained by a simple Gaussian fit. In these measurements one end of the sample was coupled to the readout device with the Dow corning 200 fluid, while all other faces of the sample were wrapped with the Tyvek paper. Since we can not distinguish the seed end from the tail end, the A end was define such that the sample produces a lower light when it was coupled to the PMT. The other end is defined as the B end.

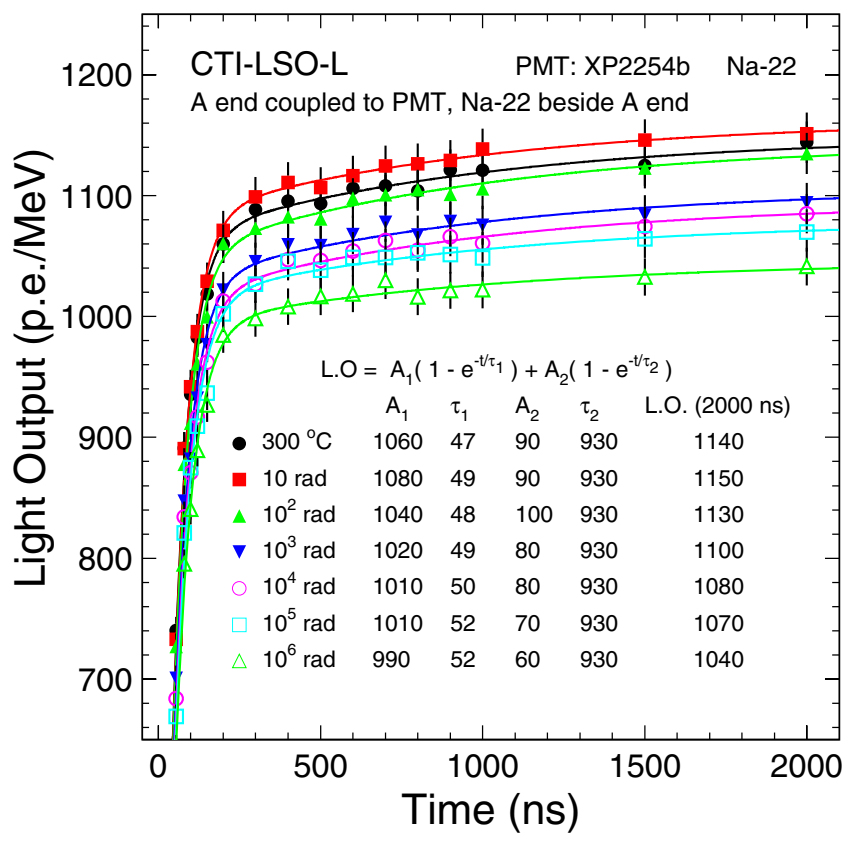

Fig. 6. Light output before and after each step of irradiations measured by using the XP2254b PMT is shown as a function of the integration time for the CTI-LSO sample.

Fig. 6 shows light output as a function of integration time for CTI-LSO with the A end coupled to the PMT. The corresponding fits for two decay components are also shown. Although there is a degradation in the light output no change in the decay kinetics was observed. The light response uniformity was measure for long samples by shooting a collimated ${ }^{22} \mathrm{Na} \gamma$ ray source at seven evenly distributed locations along crystal's 


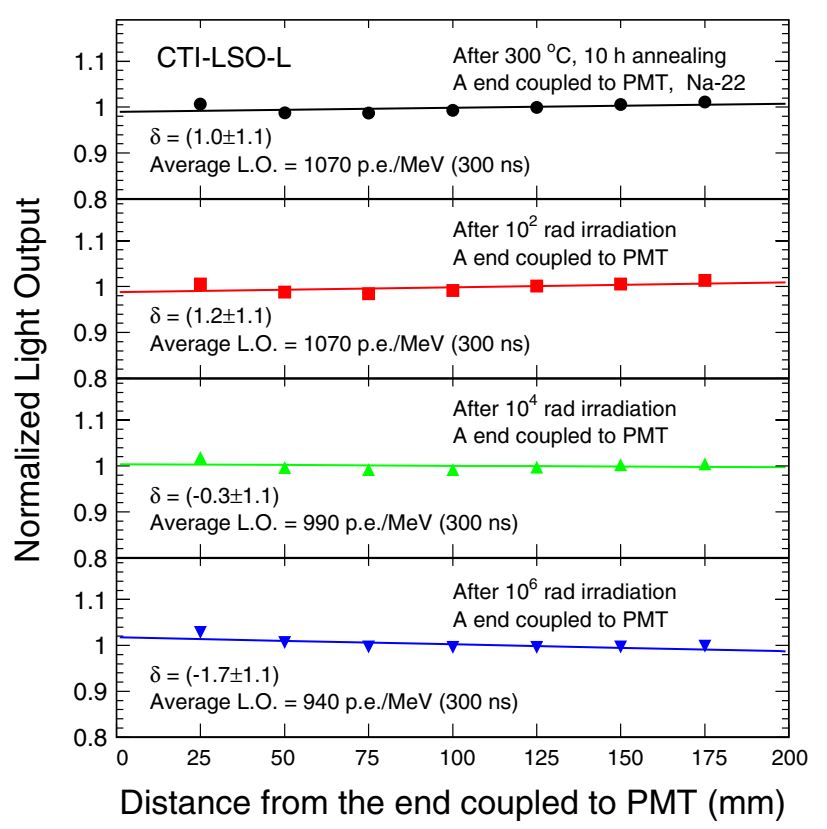

Fig. 7. Light response uniformities before and after irradiations with integrated dosage of $10^{2}, 10^{4}$ and $10^{6}$ are shown for the CTI-LSO sample with the A end coupled to the XP2254b PMT.

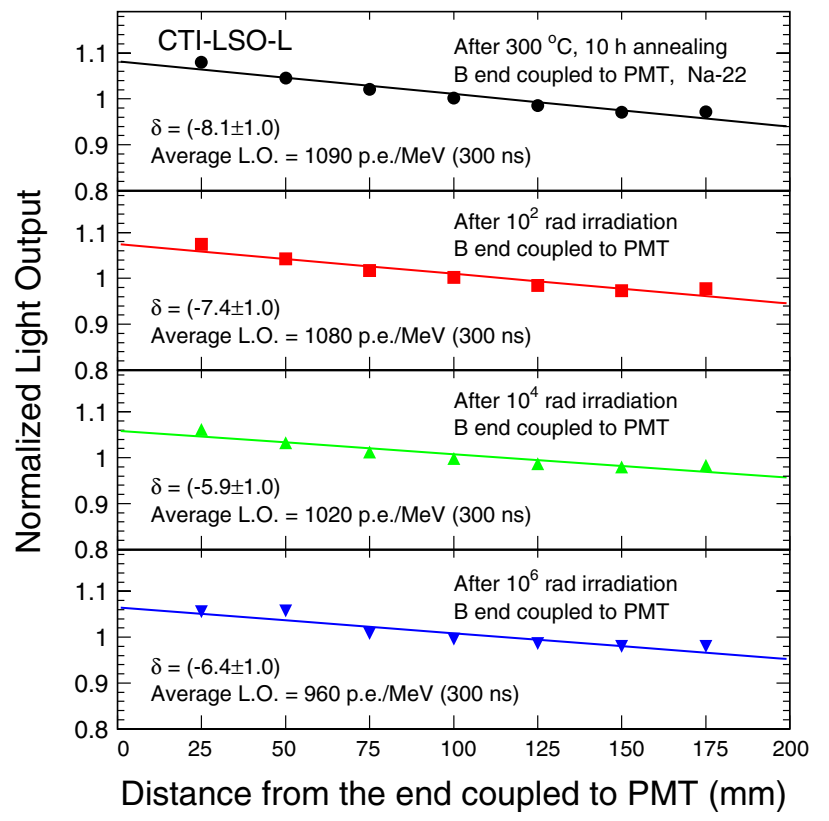

Fig. 8. The same as Fig. 7 with the B end coupled to the XP2254b PMT.

longitudinal axis with coincidence trigger. The responses at these seven points were fit to a linear function, Fig. 7 and Fig. 8 show light response uniformity for the CTI-LSO sample with A end and B end coupled to the PMT respectively.

$$
\frac{L O}{L O_{\text {mid }}}=1+\delta\left(x / x_{\text {mid }}-1\right)
$$

where $\mathrm{LO}_{\text {mid }}$ represents the light output at the middle of the sample, $\delta$ represents the deviation of the light response
TABLE II

RESUlt OF Light RESPONSE UNIFORMITY WITH PMT REAdout

\begin{tabular}{|c|c|c|c|c|c|}
\hline \multirow{2}{*}{$\begin{array}{c}\text { Sample } \\
\text { ID }\end{array}$} & \multirow{2}{*}{$\begin{array}{c}\text { Integrated } \\
\text { dosage (rad) }\end{array}$} & \multicolumn{2}{|c|}{ A end coupled } & \multicolumn{2}{|c|}{ B end coupled } \\
\hline & & $\mathrm{LO}_{\text {mid }}^{*}$ & $\delta(\%)$ & $\mathrm{LO}_{m i d}^{*}$ & $\delta(\%)$ \\
\hline \multirow{7}{*}{ CTI-LSO } & annealing & 1070 & $1 \pm 1$ & 1090 & $-8 \pm 1$ \\
\hline & 10 & 1080 & $1 \pm 1$ & 1100 & $-8 \pm 1$ \\
\hline & $10^{2}$ & 1070 & $1 \pm 1$ & 1080 & $-7 \pm 1$ \\
\hline & $10^{3}$ & 1030 & $1 \pm 1$ & 1050 & $-7 \pm 1$ \\
\hline & $10^{4}$ & 990 & $0 \pm 1$ & 1020 & $-6 \pm 1$ \\
\hline & $10^{5}$ & 980 & $-1 \pm 1$ & 1000 & $-6 \pm 1$ \\
\hline & $10^{6}$ & 940 & $-2 \pm 1$ & 960 & $-6 \pm 1$ \\
\hline \multirow{7}{*}{ CPI-LYSO } & annealing & 950 & $1 \pm 1$ & 990 & $-5 \pm 1$ \\
\hline & 10 & 970 & $0 \pm 1$ & 1000 & $-4 \pm 1$ \\
\hline & $10^{2}$ & 950 & $0 \pm 1$ & 980 & $-4 \pm 1$ \\
\hline & $10^{3}$ & 940 & $0 \pm 1$ & 930 & $-5 \pm 1$ \\
\hline & $10^{4}$ & 910 & $0 \pm 1$ & 920 & $-5 \pm 1$ \\
\hline & $10^{5}$ & 870 & $-1 \pm 1$ & 870 & $-3 \pm 1$ \\
\hline & $10^{6}$ & 850 & $-2 \pm 1$ & 850 & $-3 \pm 1$ \\
\hline \multirow{7}{*}{ SG-LYSO } & annealing & 1090 & $0 \pm 1$ & 1090 & $-5 \pm 1$ \\
\hline & 10 & 1090 & $-1 \pm 1$ & 1090 & $-5 \pm 1$ \\
\hline & $10^{2}$ & 1080 & $-1 \pm 1$ & 1080 & $-6 \pm 1$ \\
\hline & $10^{3}$ & 1050 & $-1 \pm 1$ & 1070 & $-5 \pm 1$ \\
\hline & $10^{4}$ & 1010 & $0 \pm 1$ & 1040 & $-6 \pm 1$ \\
\hline & $10^{5}$ & 940 & $0 \pm 1$ & 970 & $-6 \pm 1$ \\
\hline & $10^{6}$ & 920 & $0 \pm 1$ & 950 & $-5 \pm 1$ \\
\hline
\end{tabular}

${ }^{*} \mathrm{LO}_{\text {mid }}$ : light output at the middle of the sample (p.e./MeV).

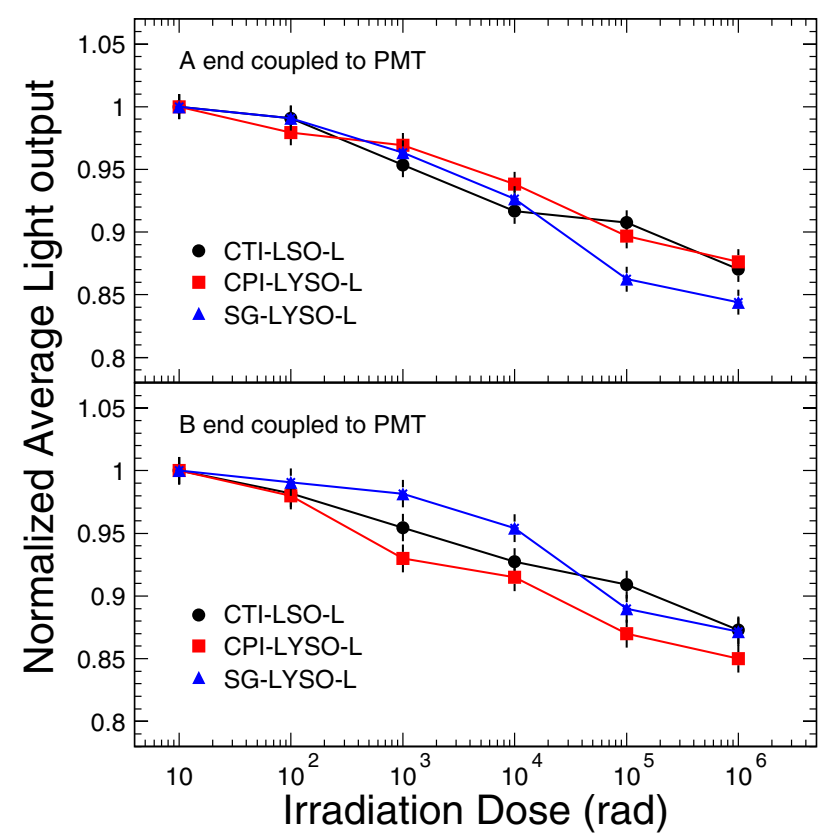

Fig. 9. Normalized light output with A (top) and B (bottom) end coupled to the XP2254b PMT is shown as a function of the integration dosage for CTI-LSO, CPI-LYSO and SG-LYSO samples.

uniformity, and $x$ is the distance from the end coupled to the 
readout device.

Table II summarizes the numerical result of light response uniformity measurements. Fig. 9 shows the degradation of the normalized average light output, $\mathrm{LO}_{\text {mid }}$, with the A (top) and B (bottom) end coupled to the PMT for all three samples after each step of irradiations. With the A end coupled to the PMT, the degradation in the average light output after $10^{6} \mathrm{rad}$ irradiation is $12 \%, 12 \%$ and $11 \%$ respectively for CIT-LSO, CPI-LYSO and SG-LYSO, while it is $12 \%, 14 \%$ and $13 \%$ for the $\mathrm{B}$ end coupled to the PMT.

The $\delta$ values with the A end coupled to the PMT are consistent with zero, which is in a good agreement with what observed in a long BGO sample [7]. The $\delta$ measured with the $\mathrm{B}$ end coupled to the PMT show relative large negative values, which may be attributed to crystal's chemical nature. It is well known that the light output of these crystals is affected by both the cerium concentration [11] and the yttrium fraction [9]. Any longitudinal variation, e.g. in Ce concentration, would affect long sample's light response uniformity. Effort has to be made to develop long crystals of consistent light response uniformity, even under irradiations [12].

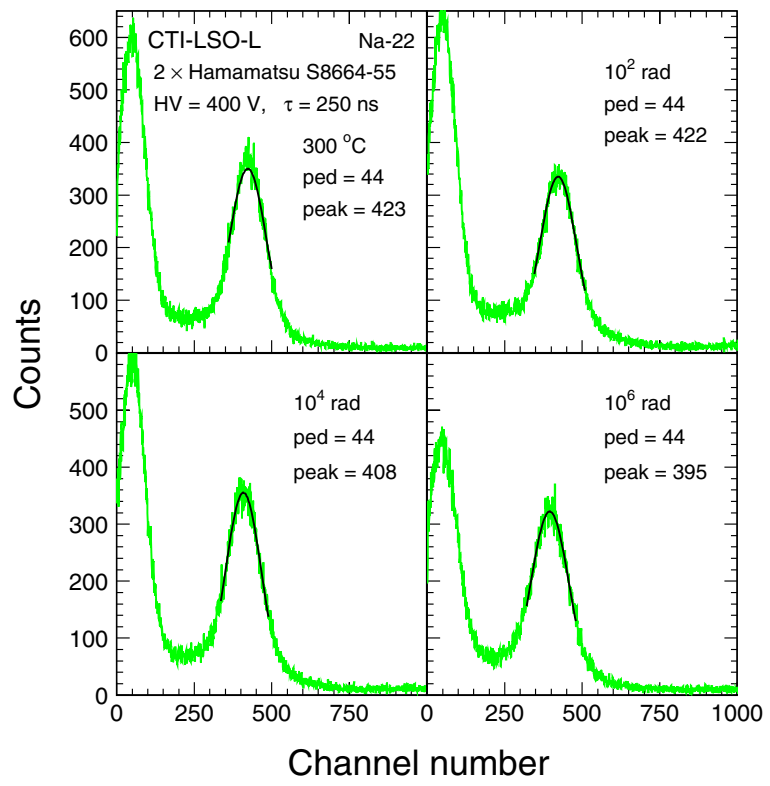

Fig. 10. The spectra of $0.511 \mathrm{MeV} \gamma$-rays from a ${ }^{22} \mathrm{Na}$ source measured using two Hamamatsu S8664-55 APD with coincidence trigger are shown for the CTI-LSO sample before and after irradiations with integrated dosage of $10^{2}, 10^{4}$ and $10^{6} \mathrm{rad}$.

\section{Light OUtPut AND RESPONSE UNIFORMity With APD READOUT}

Crystals in a high energy physics experiment are often located in a magnetic field. This excludes the use of PMT as the readout device in the most cases. Solid state devices, such as silicon photodiode (PD) or avalanche photodiode (APD), are usually used since they are immune to the magnetic field. Because of lacking sufficient amplification, this would lead to a large readout noise. For crystals with low light output,

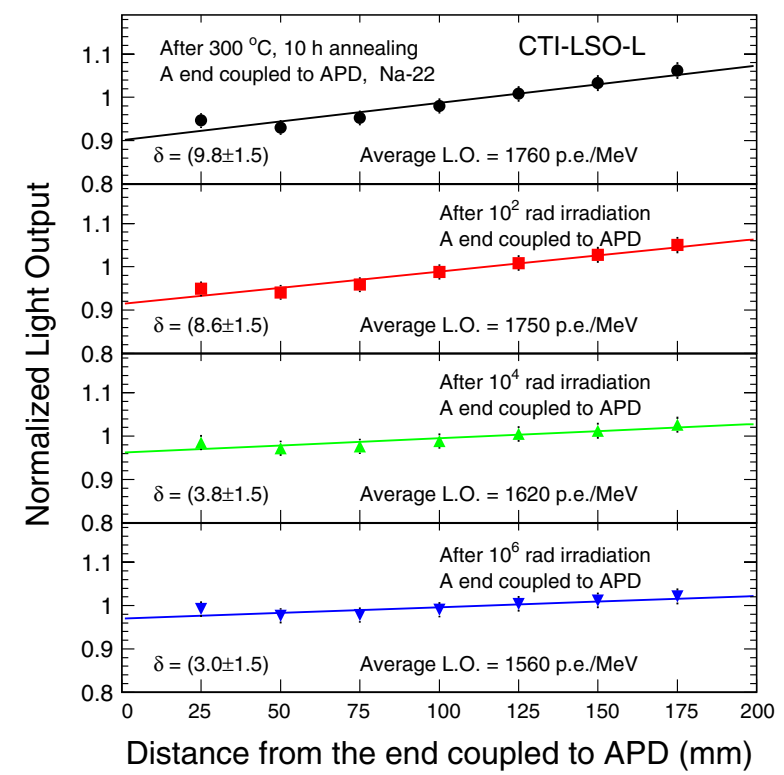

Fig. 11. Light response uniformities before and after irradiations with integrated dosage of $10^{2}, 10^{4}$ and $10^{6}$ are shown for the CTI-LSO sample with the A end coupled to the S8664-55 APDs.

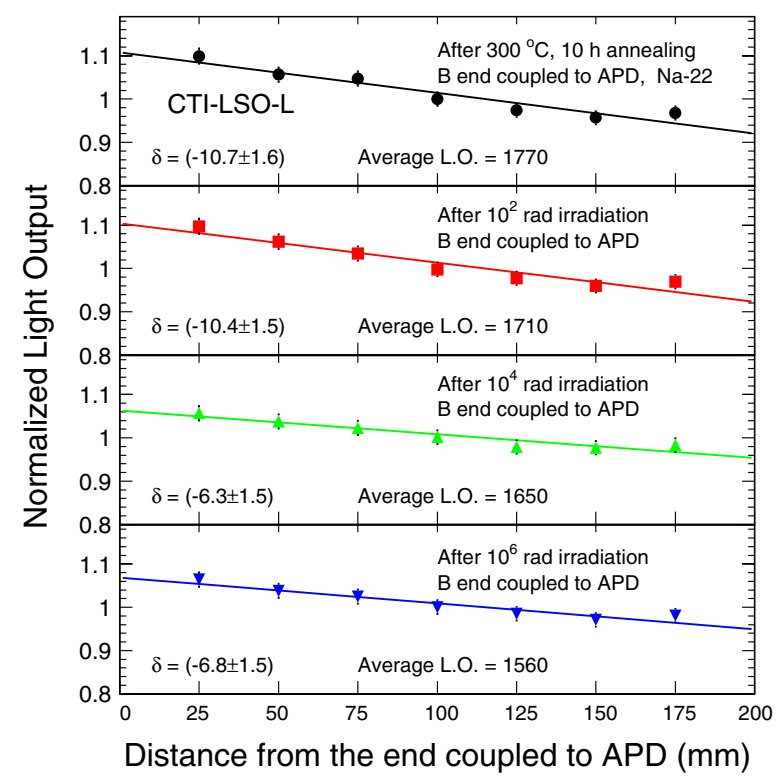

Fig. 12. Light response uniformities are shown with B end coupled to the APD for CTI-LSO sample.

laboratory measurements are often done with PMT. Because of their high light output, however, LSO and LYSO may be readout by silicon APDs in laboratory measurements [8]. Two commercial available Hamamatsu S8664-55 APDs are used as the readout device, which has a dimension of $5 \times 5 \mathrm{~mm}$. The total readout area is $0.5 \mathrm{~cm}^{2}$, corresponding to $8 \%$ coverage of crystal's back face. This low coverage was more or less compensated by the $75 \%$ quantum efficiency for LSO and LYSO scintillation light [8]. The APDs were reverse biased at $400 \mathrm{~V}$ with gain about 50 . Their output went through a 
TABLE III

RESUlt OF Light RESPONSE UNIFORMITY WITH APD READOUT

\begin{tabular}{|c|c|c|c|c|c|}
\hline \multirow{2}{*}{$\begin{array}{l}\text { Sample } \\
\text { ID }\end{array}$} & \multirow{2}{*}{$\begin{array}{c}\text { Integrated } \\
\text { dosage (rad) }\end{array}$} & \multicolumn{2}{|c|}{ A end coupled } & \multicolumn{2}{|c|}{ B end coupled } \\
\hline & & $\mathrm{LO}_{\text {mid }}^{*}$ & $\delta(\%)$ & $\mathrm{LO}_{m i d}^{*}$ & $\delta(\%)$ \\
\hline \multirow{7}{*}{ CTI-LSO } & annealing & 1760 & $10 \pm 1.5$ & 1770 & $-11 \pm 1.5$ \\
\hline & 10 & 1770 & $9 \pm 1.5$ & 1760 & $-10 \pm 1.5$ \\
\hline & $10^{2}$ & 1750 & $9 \pm 1.5$ & 1710 & $-10 \pm 1.5$ \\
\hline & $10^{3}$ & 1680 & $6 \pm 1.5$ & 1690 & $-9 \pm 1.5$ \\
\hline & $10^{4}$ & 1620 & $4 \pm 1.5$ & 1650 & $-6 \pm 1.5$ \\
\hline & $10^{5}$ & 1610 & $4 \pm 1.5$ & 1640 & $-5 \pm 1.5$ \\
\hline & $10^{6}$ & 1560 & $3 \pm 1.5$ & 1560 & $-7 \pm 1.5$ \\
\hline \multirow{7}{*}{ CPI-LYSO } & annealing & 1330 & $7 \pm 1.5$ & 1390 & $-1 \pm 1.5$ \\
\hline & 10 & 1350 & $8 \pm 1.5$ & 1390 & $-1 \pm 1.5$ \\
\hline & $10^{2}$ & 1350 & $8 \pm 1.5$ & 1370 & $-1 \pm 1.5$ \\
\hline & $10^{3}$ & 1290 & $7 \pm 1.5$ & 1330 & $1 \pm 1.5$ \\
\hline & $10^{4}$ & 1270 & $7 \pm 1.5$ & 1290 & $0 \pm 1.5$ \\
\hline & $10^{5}$ & 1260 & $7 \pm 1.5$ & 1280 & $1 \pm 1.5$ \\
\hline & $10^{6}$ & 1200 & $5 \pm 1.5$ & 1240 & $0 \pm 1.5$ \\
\hline \multirow{7}{*}{ SG-LYSO } & annealing & 1510 & $8 \pm 1.5$ & 1500 & $-5 \pm 1.5$ \\
\hline & 10 & 1520 & $7 \pm 1.5$ & 1500 & $-5 \pm 1.5$ \\
\hline & $10^{2}$ & 1520 & $7 \pm 1.5$ & 1470 & $-5 \pm 1.5$ \\
\hline & $10^{3}$ & 1500 & $7 \pm 1.5$ & 1460 & $-5 \pm 1.5$ \\
\hline & $10^{4}$ & 1440 & $7 \pm 1.5$ & 1400 & $-6 \pm 1.5$ \\
\hline & $10^{5}$ & 1410 & $7 \pm 1.5$ & 1370 & $-7 \pm 1.5$ \\
\hline & $10^{6}$ & 1370 & $6 \pm 1.5$ & 1350 & $-6 \pm 1.5$ \\
\hline
\end{tabular}

* $\mathrm{LO}_{\text {mid }}$ : light output at the middle of the sample (p.e./MeV).

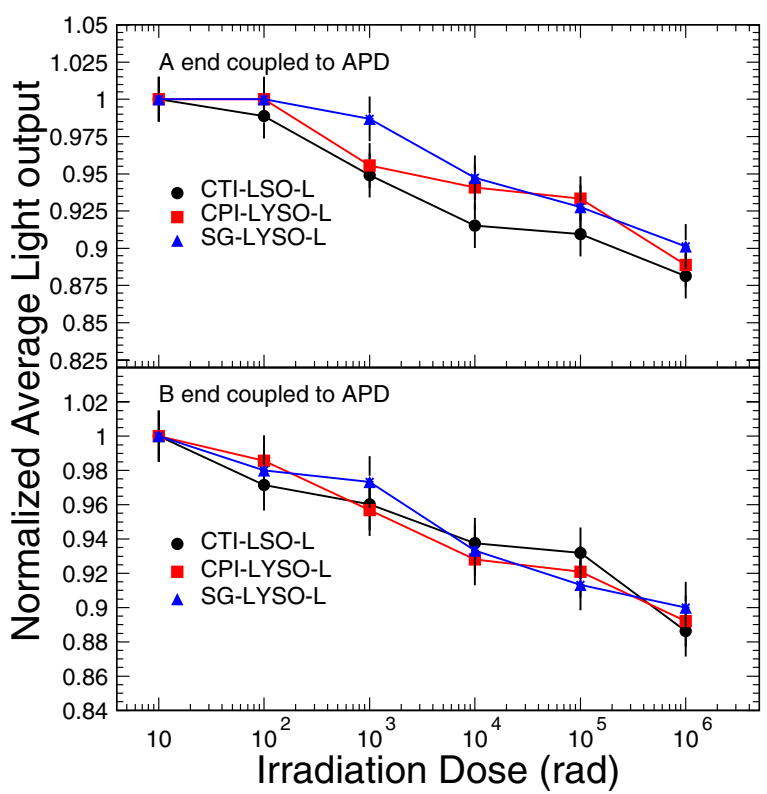

Fig. 13. Normalized light output with A (top) and B (bottom) end coupled to the S8664-55 APDs is shown as a function of the integration dosage for CTI-LSO, CPI-LYSO and SG-LYSO samples.

Canberra 2003 BT preamplifier and an ORTEC 673 shaping amplifier with shaping time set at $250 \mathrm{~ns}$.
Fig. 10 shows spectra of $0.511 \mathrm{MeV} \gamma$-rays from a ${ }^{22} \mathrm{Na}$ source measured with S8664-55 APDs with coincidence trigger for the CTI-LSO sample. Clear $\gamma$-ray peaks are visible for the sample even after an integrated dosage of $10^{6}$ rad. Fig. 11 and Fig. 12 show the light response uniformities measured by S8664-55 APDs with $0.511 \mathrm{MeV} \gamma$-rays from a ${ }^{22} \mathrm{Na}$ source and a coincidence trigger for the CTI-LSO sample with the A end and B end coupled to the APDs respectively.

Table III summarizes the numerical results. Once again, we observed end dependent uniformity slopes $\delta$ and a minor degradation of the average light output. Fig. 13 shows the corresponding degradations of the average light output measured with the A end (top) and B (bottom) end coupled to the S866455 APDs for all three samples. With the A end coupled to the APDs, the degradation in light output after $10^{6}$ rad irradiation is $11 \%, 10 \%$ and $11 \%$ respectively for CIT-LSO, CPI-LYSO and SG-LYSO, while it is $12 \%, 11 \%$ and $10 \%$ for the B end coupled to the APDs.

\section{Summary}

Three long LSO and LYSO crystal samples have gone through $\gamma$-ray irradiation up to $10^{6} \mathrm{rad}$. It was found that the shape of the photo-luminescence spectra are not affected by $\gamma$-ray irradiations, indicating that there is no damage in the scintillation mechanism. The recovery after irradiations is found to be negligible, indicating that radiation damage in LSO and LYSO crystals is not dose rate dependent. Typical light output loss is found to be at $12 \%$ level for these samples after irradiations with an integrated dosage up to $10^{6} \mathrm{rad}$. The overall radiation damage effect in LSO and LYSO crystals thus is small as compared to other commonly used crystal scintillators. In a brief summary, with existing mass production capabilities LSO and LYSO crystals are a good candidate for applications in high energy and nuclear physics.

\section{REFERENCES}

[1] K. Takagi and T. Fakazawa, Cerium activated $\mathrm{Gd}_{2} \mathrm{SiO}_{5}$ single crystal scintillator, Appl. Phys. Lett. 42 (1983) 43-45.

[2] C. Melcher and J. Schweitzer, Cerium-doped lutetium oxyorthosilicate: a fast, efficient new scintillator, IEEE Trans. Nucl. Sci. 39 (1992) 502-505.

[3] D.W. Cooke, K.J. McClellan, B.L. Bennett, J.M. Roper, M.T. Whittaker and R.E. Muenchausen, Crystal growth and optical characterization of cerium-doped $\mathrm{Lu}_{1.8} \mathrm{Y}_{0.2} \mathrm{SiO}_{5}$, J. Appl. Phys. 88 (2000) 7360-7362.

[4] T. Kimble, M Chou and B.H.T. Chai, Scintillation properties of LYSO crystals, in Proc. IEEE Nuclear Science Symposium Conference (2002).

[5] W. Wisniewski, Consideration for Calorimetry at a Super B Factory, in Proceedings of Tenth International Conference on Calorimetry in Particle Physics, Ed. R.-Y. Zhu, World Scientific (2002).

[6] R.-Y. Zhu, An LSO/LYSO Crystal Calorimeter for the ILC, talk presented in 2005 ILC Workshop, Snowmass.

[7] J.M. Chen, L.Y. Zhang and R.Y. Zhu, Large Size LYSO Crystals for Future High Energy Physics Experiments, IEEE Trans. Nucl. Sci. 52 (2005) 2133-3140.

[8] J.M. Chen, L.Y. Zhang and R.Y. Zhu, A Study on Large Size LSO and LYSO Crystals for Future High Energy Physics Experiments, paper N126 presented in 2005 IEEE NSS/MIC at Puerto Rico, will be published in IEEE Trans. Nucl. Sci. 53 (2006).

[9] B. Chai, private communication.

[10] D. Rothan, private communication.

[11] C. Melcher, private communication.

[12] R.-Y. Zhu, Radiation Damage in Scintillating Crystals, Nucl. Instr. and Meth. A413 (1998) 297-311. 